\title{
Epidural anesthesia for labor and delivery in a patient with May-Hegglin anomaly: a case report
}

This article was published in the following Dove Press journal:

Local and Regional Anesthesia

28 April 2017

Number of times this article has been viewed

\begin{abstract}
Annas Muhammad
Muzannara

Mohammed Al Harbi

Raza Rathore

Nasser Tawfeeq

Freddie Wambi

Nasir Mahmood

Sonia Albrechtova

Vassilios Dimitriou

Department of Anesthesia, King Abdulaziz Medical City, National Guard Health Affairs, Riyadh, Kingdom of Saudi Arabia
\end{abstract}

Correspondence: Vassilios Dimitriou Department of Anesthesia, King Abdulaziz Medical City, National Guard Health Affairs, Riyadh I46II, Kingdom of Saudi Arabia

Email vaskdimi58@gmail.com
Abstract: We report a case of May-Hegglin anomaly (MHA) in a woman who had a successful labor and delivery under epidural anesthesia. MHA is an inherited thrombocytopenia easily misdiagnosed as idiopathic (immune) thrombocytopenic purpura (ITP). Early and appropriate diagnosis of MHA during pregnancy is essential for optimal maternal and neonatal delivery outcome. Additionally, it can avoid unnecessary diagnostic studies, such as bone marrow aspiration and biopsy, and even harmful therapies with corticosteroids, immunosuppressive agents, and splenectomy. Consequently, the most serious impacts of this disease are iatrogenic managements due to misdiagnosis. It seems that in patients with MHA, adequate clinical coagulation is far more dependent on adequate platelet function than any particular platelet count. The diagnosis of MHA may pose a challenge for clinicians managing pregnant women with thrombocytopenia. Keywords: May-Hegglin anomaly, thrombocytopenia, pregnancy

\section{Introduction}

May-Hegglin anomaly (MHA) is a rare hematological disorder, inherited as an autosomal dominant trait, within the family of myosin heavy chain (MHC) disorders, including Fechtner syndrome, Sebastian syndrome, Epstein syndrome, and Alport-like syndromes. ${ }^{1-4}$ This group is also known as MYH9-related disorders (MYH9RDs), because all have largely overlapping phenotypes and result from mutations in the MYH9 gene on chromosome 22, which encodes the nonmuscle myosin heavy chainIIA (NMMHC-IIA) protein. ${ }^{3-5}$ NMMHC-IIA is a cytoplasmic protein with expression in many tissues, including platelets. ${ }^{1-4}$

MYH9RDs are characterized by varying degrees of thrombocytopenia, giant platelets, and large $(2-5 \mu \mathrm{m})$, well-defined, basophilic, cytoplasmic inclusion bodies (resembling Döhle bodies) in the granulocytes. ${ }^{1-5}$ These inclusion bodies result from deposition of MHC within white blood cells. Interestingly, they are not seen in platelets, and their presence in leukocytes can differentiate MHA from idiopathic (immune) thrombocytopenic purpura (ITP). ${ }^{1-5}$

\section{Top of Form}

MYH9RDs are considered very rare. The Italian Registry for MYH9RD indicates that the prevalence of the disorder in Italy is at least $3: 1,000,000 .{ }^{6}$ Because mild forms are discovered incidentally and severe forms are often misdiagnosed or underreported as other disorders, the actual prevalence is expected to be higher. MYH9RD has been diagnosed worldwide, and there is no evidence of variation in prevalence across ethnic 
populations. ${ }^{7}$ In general, the worldwide incidence of MHA is unknown.

Thrombocytopenia occurs in approximately $50 \%$ of the patients with MHA, and the clinical manifestations vary from mild bleeding not requiring specific treatment up to severe bleeding episodes following trauma or surgery that require blood products. ${ }^{1-3,8}$ However, despite severe thrombocytopenia in most patients, platelet function is frequently normal. Therefore, they are asymptomatic, discovered incidentally. ${ }^{1-3,8}$

Thrombocytopenia is common during pregnancy; however, MHA is very rare, with 40 cases reported in the literature. ${ }^{9}$ Diagnosis has been established prior to pregnancy in some women, but in the majority, the problem is first identified incidentally during pregnancy as thrombocytopenia on routine booking blood tests. Most of such cases, without careful inspection of blood smears and a thorough family and bleeding history, are initially misdiagnosed as refractoryto-treatment ITP. ${ }^{9}$ Consequently, the most serious impacts of this disease are iatrogenic managements due to misdiagnosis. ${ }^{3}$ The diagnosis of MHA may pose a challenge for clinicians managing pregnant women with thrombocytopenia. Herein, we report a case of MHA in a woman who had a successful labor and delivery under epidural anesthesia. The patient provided written informed consent for her data to be included in this case report.

\section{Case description}

The history of the patient starts 10 years ago on April 2006, when she presented as primigravida at 36 weeks gestation, booked for antenatal investigations. Patient was not known to have any medical illness or history of previous surgical procedures. Her platelet count was found to be $22 \times 10^{9} / \mathrm{L}$, and she was admitted as a case of possible ITP. Coagulation profile was within normal limits, and there was no history of bleeding tendency. Family history positive for bleeding disorders was not reported. She was started on steroid therapy (initially with prednisolone and later with dexamethasone); however, the platelet count decreased to $19 \times 10^{9} / \mathrm{L}$. After transfusion of 6 units of platelets, her platelet count was $20 \times 10^{9} / \mathrm{L}$. Then, she received four cycles of rituximab (monoclonal antibody) and three doses of immunoglobulin, without response. Bone marrow biopsy revealed thalassemia trait and iron deficiency. Megakaryocytes and erythroblasts exhibited significant dysplasia. Differential diagnosis was between ITP and myelodysplastic syndrome. She had an uncomplicated cesarean section under general anesthesia, due to failure to labor progress. She received 18 units of platelets and 6 units of cryoprecipitate since the cesarean section. No bleeding tendency was observed perioperatively. Postoperatively, her platelet count was $65 \times 10^{9} / \mathrm{L}$, with normal coagulation profile. She was discharged without any complication.

After 3 months, a second bone marrow biopsy did not reveal additional pathology and confirmed the initial diagnosis (ITP). Her platelet count was $18 \times 10^{9} / \mathrm{L}$, so the patient was scheduled for splenectomy due to refractory ITP. In August 2006, she was transfused with 8 units of platelets and underwent an uncomplicated laparoscopic splenectomy under general anesthesia, with minimal blood loss. The spleen pathology report was consistent with the clinical diagnosis of ITP. After splenectomy, she had a platelet count $8 \times 10^{9} / \mathrm{L}$. Two months later, she showed improvement to $50 \times 10^{9} / \mathrm{L}$ and, at 6 months, to $100 \times 10^{9} / \mathrm{L}$. However, then her platelet count decreased again and ranged between 7 and $20 \times 10^{9} / \mathrm{L}$.

In December 2007, the patient was then evaluated while she was on her second pregnancy at 2 months' gestation. Her platelet count was $7 \times 10^{9} / \mathrm{L}$, and she was advised admission and the need for treatment. However, she signed against medical advice. She was transfused with 6 units of platelets and received intravenous (IV) immunoglobulin $55 \mathrm{~g}(1 \mathrm{~g} / \mathrm{kg})$ and then was discharged with platelet count $45 \times 10^{9} / \mathrm{L}$ and on oral treatment with prednisolone. In June 2008, she had an uncomplicated labor and delivery that did not need any anesthetic management. During regular follow-up evaluations, the patient presented with hypothyroidism and started treatment with oral thyroxine.

About 2 years later, in January 2011, during regular follow-up evaluations, microscopic assessment of a peripheral blood smear showed giant platelets and cytoplasmic inclusion bodies in the granulocytes. Therefore, the patient was diagnosed as suffering from MHA. Hemoglobin electrophoresis revealed findings consistent with alpha thalassemia trait and sickle cell trait with iron deficiency anemia. The patient had another bone marrow examination, which revealed that she did not have ITP and that her thrombocytopenia was due to $M Y H 9$ gene-related hereditary macrothrombocytopenia. During her further course, she had two more pregnancies in 2011 and 2012, with spontaneous abortions. Her platelet count ranged between 8 and $20 \times 10^{9} / \mathrm{L}$.

In 2014, the patient presented for labor and delivery after her fifth pregnancy, with spontaneous rupture of membrane at 39 weeks' gestation. Her platelet count was $13 \times 10^{9} / \mathrm{L}$, and she was transfused with 6 units of platelets, which increased the platelet count to $24 \times 10^{9} / \mathrm{L}$. The hematology consult was that the patient should be treated as a patient with a normal coagulation status and did not require any other specific treatment. Additionally, a neuraxial blockade 


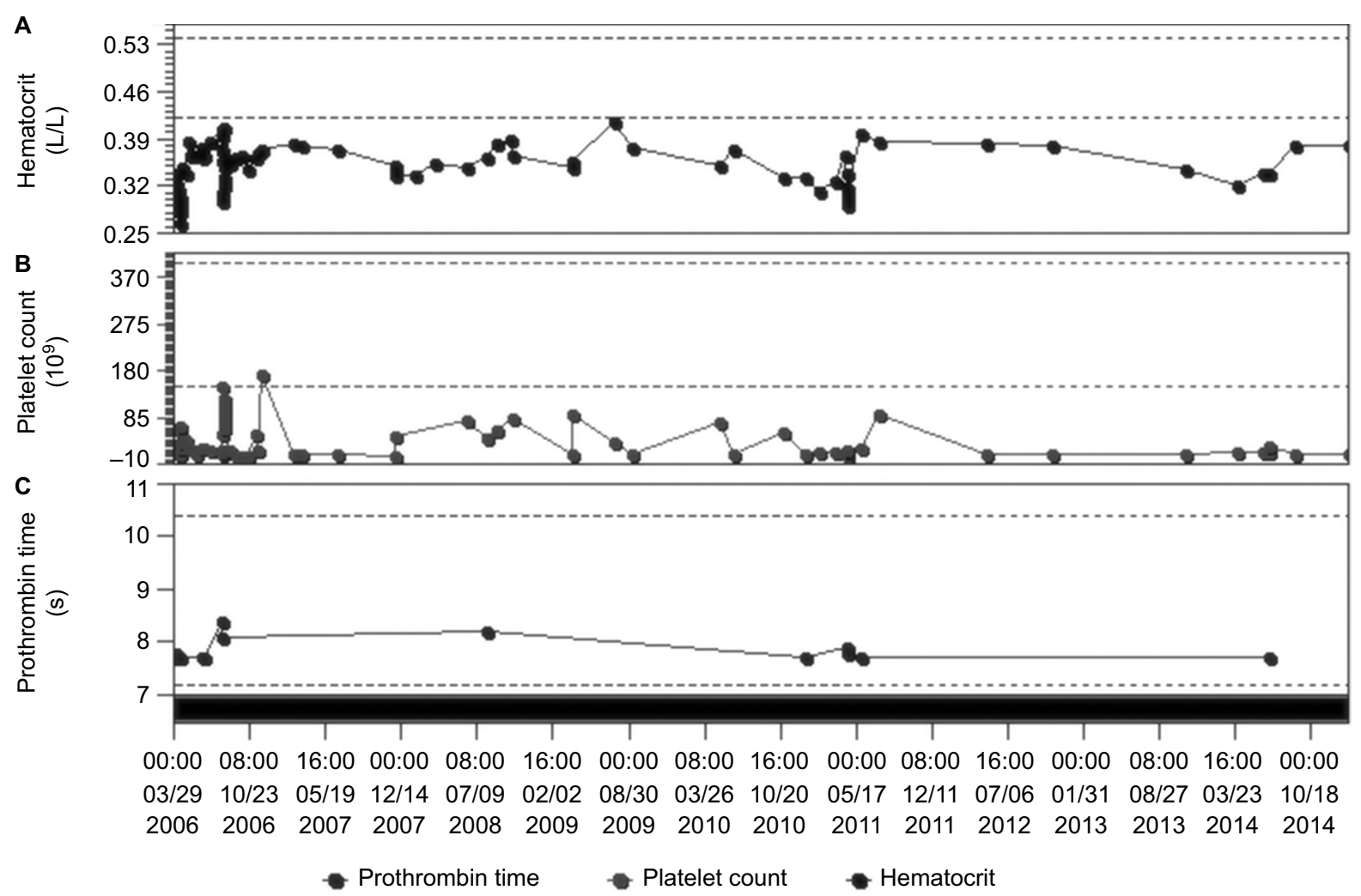

Figure I Graphic representation of a period of 9 years (2006-2014), presenting the range of changes in (A) hematocrit, (B) platelet count, and (C) prothrombin time. Notes: Platelet count was transiently increased only after massive platelet transfusions, while most of the time, it ranged between 10 and $20 \times 10^{9} / \mathrm{L}$. At the same time, no significant changes were noted in prothrombin time and hematocrit, except during labor and delivery.

could be performed if needed. Once requested, an epidural catheter was easily inserted at L1-L2. The patient had an uncomplicated delivery and an uneventful hospital stay. She was discharged after 6 days.

Figure 1 shows a graphic over a period of 9 years (20062014), presenting the range of changes in platelet count, prothrombin time, and hematocrit of the patient. Platelet count was transiently increased only after massive platelet transfusions, while most of the time, it ranged between 10 and $20 \times 10^{9} / \mathrm{L}$. At the same time, no significant changes were noted in prothrombin time and hematocrit, except during labor and deliveries.

\section{Discussion}

We have presented a case of MHA in a woman who underwent a successful labor and delivery under epidural anesthesia. Review of literature revealed that neuraxial blockade has been rarely reported in the past in MHA cases. Spinal anesthesia for cesarean section has been applied in four cases and epidural analgesia applied for labor and delivery in five cases. ${ }^{10-13}$

Interestingly, there is still no consensus regarding the absolute lower limit of platelet count that confers safety for neuraxial anesthesia. Even the very recent (2016) guidelines for obstetrical anesthesia from the American Society of Anesthesiologists (ASA) ${ }^{14}$ avoid suggesting a clear guidance and just state: "A specific platelet count predictive of neuraxial anesthetic complications has not been determined." In general, a platelet count of $\geq 50 \times 10^{9} / \mathrm{L}$ is recommended for safe delivery. ${ }^{4}$ Therefore, in most cases, general anesthesia is preferred, due to severe thrombocytopenia and considering the risk of possible spinal or epidural hematoma. ${ }^{14-16}$

MYH9RDs result from mutations in the MYH9 gene. Depending on the position of the causative mutation within the gene, the risk increases for syndromic clinical manifestations., ${ }^{3,17}$ Bleeding diathesis, high-tone hearing loss, glomerular nephropathy, and presenile cataract are the clinical features of MYH9RDs in descending order of frequency. ${ }^{5}$ Mutations in the neck region of the NMMHC-IIA protein are more likely associated with these comorbidities than mutations in the $\mathrm{N}$ - or C-terminal part of the gene., ${ }^{3,17}$ To date, at least 49 mutations of the MYH9 gene have been identified. ${ }^{6}$ More specifically, MHA is caused by abnormalities in the MYH9 gene located on chromosome 22q12-13 and encoding NMMHC-IIA. ${ }^{1-4}$ The diagnosis of MHA has been conventionally based on morphological criteria alone, 
by microscopic assessment of a peripheral blood smear after conventional staining (such as May-Grünwald-Giemsa staining), demonstrating a triad of giant platelets, thrombocytopenia, and inclusions in the cytoplasm of leukocytes. ${ }^{1-4}$ It is important to note that electronic cell counters work mainly based on the size and therefore they often classify very large platelets as erythrocytes. As a consequence, in MYH9RD, these instruments greatly underestimate mean platelet volume, as well as platelet count..$^{18,19}$

The pathogenesis of MHA is poorly understood. Reported platelet counts in MHA have ranged from less than $10 \times 10^{9} / \mathrm{L}$ to normal levels, indicating variable expressivity; however, the platelet structure, function, and life span are usually normal. ${ }^{20}$ Additionally, no correlation was found between bleeding tendency and platelet count. ${ }^{2}$ A qualitative defect of platelets may be responsible for mild bleeding diathesis even in the absence of thrombocytopenia, while severe bleeding results from both qualitative and quantitative platelet defects. Bone marrow examination shows normal megakaryocytes. The cause of the thrombocytopenia in MHA is thought to be defective megakaryocyte maturation and fragmentation, which may account for production of large platelets. ${ }^{21}$

Thrombocytopenia is common during pregnancy. The most common causes include gestational thrombocytopenia, preeclamptic disorders of pregnancy and ITP. However, MHA is rare during pregnancy. ${ }^{9}$ Whenever a family history of thrombocytopenia is absent or unclear, evaluation of peripheral blood slides is a simple and effective tool to distinguish patients with MHA from those with ITP, as platelets are significantly larger in patients with MHA than in those with ITP. In particular, a mean platelet diameter $>3.74 \mu \mathrm{m}$ distinguishes MHA from ITP with $86 \%$ sensitivity and $87 \%$ specificity. ${ }^{22}$

The clinical spectrum of congenital thrombocytopenias in women ranges from severe bleeding tendency, recognized within the first few weeks of life, to mild conditions that may remain undetected even in adulthood. Positive bleeding history may include heavy menstrual bleeding, easy bruising, nose bleedings, bleeding after tooth extraction, and gum bleeding. Clinically, most women with MHA are asymptomatic, discovered incidentally, and require no specific treatment. In a recent systematic review of MHA during pregnancy, it was reported that $33 \%$ of women had no history of any bleeding symptoms. ${ }^{9}$

As the MHA is an autosomal dominant characteristic, the fetus has $50 \%$ chance of inheriting the pathogenic variant and presenting thrombocytopenia and MHA. ${ }^{1-5,23}$ Therefore, vaginal deliveries in women with severe thrombocytopenia should be considered at increased risk for neonatal intracranial bleeding. ${ }^{1,4}$ Approximately $65 \%$ of individuals diagnosed with MYH9RD have an affected parent. ${ }^{3-5}$ Prenatal diagnosis for pregnancies at increased risk is possible if the pathogenic variant in the family is known. ${ }^{4}$ The definitive diagnosis of MHA is limited to those with inclusion bodies in granulocytes, due to aggregates of abnormal NMMHCIIA, and no other organ dysfunction. ${ }^{8}$ Genetic analysis can be used if the evidences are nondiagnostic, but access to this test is generally only available through specialist molecular testing facilities.

Differentiating between inherited and acquired thrombocytopenias may be difficult, and significant numbers of patients with MHA have been misdiagnosed with refractory ITP. $^{3,24,25}$ In most cases, the first presentation is identified during pregnancy as thrombocytopenia during routine antenatal blood tests, with incidental finding of low platelet count. The biggest risk for these patients is that they are subjected to unnecessary, ineffective, and potentially harmful treatments based on the misdiagnosis of ITP. ${ }^{3}$ Consequently, the most serious impacts of this disease are iatrogenic managements due to misdiagnosis, and many of such patients suffer a lot before the final MHA diagnosis is established. Like in our case, a common scenario includes ineffective immunosuppressive (steroids) and intravenous immunoglobulin-G treatment, unnecessary repeated platelet and cryoprecipitate transfusions, multiple painful bone marrow examinations, and eventually ineffective, unnecessary, and potentially harmful splenectomy for steroid-resistant ITP. ${ }^{26,27}$

Routine transfusions of platelet concentrates are used in patients with MHA, to transiently increase platelet count. However, this is unnecessary and carries associated risks of possible alloimmunization, producing antibodies against human leukocyte antigen (HLA) and subsequent refractoriness to platelet infusions. ${ }^{3-5}$ Therefore, platelets and blood should be ready available for use; however, they should be administered only if abnormal active bleeding occurs, which cannot be otherwise managed. ${ }^{4,28}$ When available, transfusing platelets from HLA-matched donors should be preferred, as this reduces the risk of alloimmunization., ${ }^{4,28}$

There is no known prevention or treatment for the nonhematopoietic consequences of MHA. Previous therapy with steroids and/or high-dose immunoglobulins had no effect on platelet count or bleeding diathesis..$^{3-5,9}$ Hemostatic prophylaxis for labor and delivery may include desmopressin $\left(\right.$ DDAVP $\left.^{\circledR}\right)$, tranexamic acid, and cryoprecipitate transfusion. ${ }^{4,29}$ Preoperative use of desmopressin can be considered, because it has been proven to reduce the bleeding tendency in many acquired and congenital disorders of platelet function, including MHA. ${ }^{4,8,29}$ An assessment of the bleeding risk 
should be performed during the third trimester of pregnancy. Appropriate hemostatic cover can then be arranged to minimize the risk of primary postpartum hemorrhage. Women with no bleeding history and platelet count of at least $80-109 \times 10^{9} / \mathrm{L}$ are at low risk for bleeding and do not require platelet transfusion for labor and delivery. ${ }^{4}$ Desmopressin and/or tranexamic acid are generally adequate as hemostatic cover. ${ }^{4,29}$ However, women with a positive bleeding history or with platelet count less than $50 \times 10^{9} / \mathrm{L}$ may additionally require prophylactic platelet transfusion. Eventually, splenectomy, which is a treatment for refractory ITP, has not shown any improvement in the platelet count or any decrease in the bleeding tendency. ${ }^{26,27}$ Consequently, splenectomy is contraindicated in all hereditary macrothrombocytopenias, including MHA. ${ }^{26,27}$

\section{Conclusion}

MHA is an inherited thrombocytopenia that is easily misdiagnosed as ITP, if careful inspection of blood smear and family history are overlooked. Early and appropriate diagnosis of MHA during pregnancy is essential for optimal maternal and neonatal delivery outcome. Additionally, this can avoid unnecessary diagnostic studies, such as bone marrow aspiration and biopsy, and even harmful therapies with corticosteroids, immunosuppressive agents, and splenectomy. It seems that in patients with MHA, adequate clinical coagulation is far more dependent on adequate platelet function than any particular platelet count. A qualitative defect of platelets may be responsible for mild bleeding diathesis even in the absence of thrombocytopenia, while severe bleeding results from both qualitative and quantitative platelet defects. MHA should be suspected whenever a patient has a low platelet count or a bleeding diathesis of unknown origin. Early and appropriate diagnosis of MHA during pregnancy is essential for optimal maternal and neonatal delivery outcome.

\section{Disclosure}

The authors report no conflict of interest in this work.

\section{References}

1. Saito H, Kunishima S. May-Hegglin anomaly. Am J Hematol. 2008;83: 304-306.

2. Noris P, Spedini P, Belletti S, Magrini U, Balduini CL. Thrombocytopenia, giant platelets, and leukocyte inclusion bodies (May-Hegglin anomaly): clinical and laboratory findings. Am J Med. 1998;104(4):355-360.

3. Althaus K, Greinacher A. MYH9-related platelet disorders. Semin Thromb Hemost. 2009;35(2):189-203.

4. Savoia A, Pecci A. MYH9-related disorders. GeneReviews ${ }^{\circledR}$ [Internet] [updated July 16, 2015]. Available from https://www.ncbi.nlm.nih.gov/ books/NBK2689/. Accessed January 12, 2017.

5. Balduini CL, Pecci A, Savoia A. Recent advances in the understanding and management of MYH9-related inherited thrombocytopenias. $\mathrm{Br} J$ Haematol. 2011;154(2):161-174.
6. Saposnik B, Binard S, Fenneteau O, et al; French MYH9 networka. Mutation spectrum and genotype-phenotype correlations in a large French cohort of MYH9-related disorders. Mol Genet Genomic Med. 2014;2(4):297-312.

7. Registro Italiano della Malattia MYH9-correlata [homepage on the Internet]. [updated June 2014]. Available from: http://www.registromyh9.org/. Accessed January 12, 2017.

8. Sehbai AS, Abraham J, Brown VK. Perioperative management of a patient with May-Hegglin anomaly requiring craniotomy. Am J Hematol. 2005;79(4):303-308.

9. Hussein BA, Gomez K, Kadir RA. May-Hegglin anomaly and pregnancy: a systematic review. Blood Coagul Fibrinolysis. 2013;24(5):554-561.

10. Fishman EB, Connors JM, Camann WR. Anesthetic management of seven deliveries in three sisters with the May-Hegglin anomaly. Anesth Analg. 2009;108(5):1603-1605.

11. Duff P, Jackson MT. Pregnancy complicated by rhesus sensitization and the May-Hegglin anomaly. Obstet Gynecol. 1985;65(3 suppl):7S-10S.

12. Kotelko DM. Anaesthesia for caesarean delivery in a patient with MayHegglin anomaly. Can J Anaesth. 1989;36(3 pt 1):328-330.

13. Nelson LH, Dewan DM, Mandell GL. Obstetric and anesthetic considerations in the May-Hegglin anomaly. A case report. J Reprod Med. 1993;38(4):311-313.

14. Practice guidelines for obstetric anesthesia: an updated report by the American Society of Anesthesiologists Task Force on Obstetric Anesthesia and the Society for Obstetric Anesthesia and Perinatology. Anesthesiology. 2016;124:270-300.

15. Takabayashi R, Nishikido O, Nagano K, et al. Anesthetic management for cesarean delivery in a patient with May Hegglin anomaly. Masui. 2007;56:1198-1199.

16. Garcia Vallejo G, Cabellos M, Kabiri M, Fraile JR, Cuesta J. Anaesthetic implications in a pregnant patient with an extreme thrombocytopenia due to a May-Hegglin anomaly: general or regional anaesthesia? Rev Esp Anestesiol Reanim. 2014;61:460-465.

17. Pecci A, Panza E, Pujol-Moix N, et al. Position of nonmuscle myosin heavy chain IIA (NMMHC-IIA) mutations predicts the natural history of MYH9-related disease. Hum Mutat. 2008;29(3):409-417.

18. Althaus K, Greinacher A. MYH9-related platelet disorders. Semin Thromb Hemost. 2009;35(2):189-203.

19. Urato AC, Repke JT. May-Hegglin anomaly: a case of vaginal delivery when both mother and fetus are affected. Am J Obstet Gynecol. 1998; 179(1):260-261.

20. Hamilton RW, Shaikh BS, Ottie JN, et al. Platelet function, ultrastructure and survival in the May-Hegglin anomaly. Am J Clin Pathol. 1980; 74:663-668

21. Goodwin HA, Ginsburg AD. May-Hegglin anomaly: a defect in megakaryocyte fragmentation? Br J Haematol. 1974;26:117-127.

22. Noris P, Biino G, Pecci A, et al. Platelet diameters in inherited thrombocytopenias: analysis of 376 patients with all known disorders. Blood. 2014;124(6):e4-e10.

23. Seri M, Pecci A, Di Bari F, et al. MYH9-related disease: May-Hegglin anomaly, Sebastian syndrome, Fechtner syndrome, and Epstein syndrome are not distinct entities but represent a variable expression of a single illness. Medicine. 2003;82:203-215.

24. Balduini CL, Noris P. Inherited thrombocytopenias. Hematology. 2015;20:374-375.

25. Balduini CL, Pecci A, Noris P. Diagnosis and management of inherited thrombocytopenias. Semin Thromb Hemost. 2013;39(2):161-171.

26. Drachman JG. Inherited thrombocytopenia: when a low platelet count does not mean ITP. Blood. 2004;103(2):390-398.

27. Balduini CL, Drachman JG. Role of splenectomy in inherited thrombocytopenias. Blood. 2004;104:1227.

28. Chabane H, Gallais Y, Pathier D, Tchernia G, Gaussem P. Delivery management in a woman with thrombocytopenia of the May-Hegglin anomaly type. Eur J Obstet Gynecol Reprod Biol. 2001;99: 124-125.

29. Rosen AC, Hafner E, Sternsite W, Kier P, Kyrle A. Das May-HegglinSyndrom in der Schwangerschaft. [Das May-Hegglin-Syndrom in der Schwangerschaft]. Geburtshu Frauenheilk. 1997;57:301-312. German. 


\section{Publish your work in this journal}

Local and Regional Anesthesia is an international, peer-reviewed, open access journal publishing on the development, pharmacology, delivery and targeting and clinical use of local and regional anesthetics and analgesics. The journal is included in PubMed, and welcomes submitted papers covering original research, basic science, clinical studies, reviews and evaluations, guidelines, expert opinion and commentary, case reports and extended reports. The manuscript management system is completely online and includes a very quick and fair peer-review system, which is all easy to use. Visit http://www.dovepress.com/ testimonials.php to read real quotes from published authors.

Submit your manuscript here: https://www.dovepress.com/local-and-regional-anesthesia-journal 